\title{
Approaching perfect microwave photodetection in circuit QED
}

\author{
B. Peropadre, ${ }^{1}$ G. Romero, ${ }^{2}$ G. Johansson, ${ }^{3}$ C. M. Wilson, ${ }^{3}$ E. Solano,,${ }^{2,4}$ and J. J. García-Ripoll ${ }^{1}$ \\ ${ }^{1}$ Instituto de Física Fundamental, CSIC, Calle Serrano 113-bis, E-28006 Madrid, Spain \\ ${ }^{2}$ Departamento de Química Física, Universidad del País Vasco-Euskal Herriko Unibertsitatea, Apartado 644, E-48080 Bilbao, Spain \\ ${ }^{3}$ Department of Microtechnology and Nanoscience, Chalmers University of Technology, Göteborg, Sweden \\ ${ }^{4}$ IKERBASQUE, Basque Foundation for Science, Alameda Urquijo 36, E-48011 Bilbao, Spain
}

(Received 12 January 2011; published 14 December 2011)

\begin{abstract}
In order to apply all ideas from quantum optics to the field of quantum circuits, one of the missing ingredients is a high-efficiency single-photon detector. In this work we propose a design for such a device which successfully reaches $100 \%$ efficiency with only one absorber. Our photon detector consists of a three-level system (a phase qubit) coupled to a semi-infinite one-dimensional waveguide (a microwave transmission line) which performs highly efficient photodetection in a simplified manner as compared to previous proposals. Using the tools of quantum optics we extensively study the scattering properties of realistic wave packets against this device, thereby computing the efficiency of the detector. We find that the detector has many operating modes, can detect detuned photons, is robust against design imperfections, and can be made broadband by using more than one absorbing element in the design. Many of these ideas could be translated to other single-mode photonic or plasmonic waveguides interacting with three-level atoms or quantum dots.
\end{abstract}

DOI: 10.1103/PhysRevA.84.063834

PACS number(s): 42.50.Pq, 85.25.Pb, 85.60.Gz

\section{INTRODUCTION}

The field of quantum circuits is an interdisciplinary one which combines ideas and tools from quantum optics with the novel possibilities brought by superconducting circuits. This field is undergoing a silent revolution, which started with the first superconducting qubits [1-4], greatly advanced in the matter-wave interaction field [5-7], and is now preparing the foundations of an entirely new technology: propagating quantum microwaves. The first ingredients in this new field are the generation of nonclassical propagating waves-either through qubits and cavities [8,9], or through nonlinearities [10] — and the analysis of those fields, currently done using quantum homodyne detection techniques $[8,9,11-13]$. In order to consolidate and complete the field, we still lack two other ingredients: photon-photon interactions and single-shot photon detection and counting. In particular, photodetection is the ultimate and most desired goal. It is common to quantum optics and quantum-information protocols, from trivial homodyne detection methods up to sophisticated alloptical quantum-computing protocols [14]. Developing such a tool in circuit QED would open the door to quantum communication, quantum cryptography, and general-purpose quantum-information processing with propagating photons. In short, circuit QED allows quantum opticians to explore novel physics and technologies that are not yet available for real atoms interacting with electromagnetic fields.

In previous work $[15,16]$, we identified photodetectors as the ultimate missing tool in circuit QED, and helped in specifying the desired properties of such a device: it should be single shot, work outside the cavity [17], achieve great efficiency, be broadband, and be passive. In that same work, we proposed a rather minimal design that performed the task $[15,16]$ : coupling phase qubits to open transmission lines. In our design the phase qubit acts as a metastable three-level system which can absorb individual photons from the one-dimensional photonic waveguide and transition into a third, easily detectable state, in a process that implements single-photon detection [Fig. 1(b)] with strict upper limit of $50 \%$. We showed that by adding more qubits this value could be easily increased up to $100 \%$. This also had the side effect of improving both the bandwidth and robustness of the detector.

In this work, we show that a slight modification of our design boosts its efficiency up to $100 \%$ for a single-qubit detector, without affecting the bandwidth or robustness of the original design. The small change consists of embedding the three-level system in a semi-infinite line, at some distance from the end, which behaves as a perfect mirror. Qualitatively, in this new setup the end mirror allows incoming photons to bounce back from the end of the line and have several chances to be detected just by a single qubit. Alternatively, the setup can be seen as a one-dimensional implementation of the idea in Ref. [18], by which a two-level system is made to absorb a photon whose wave function is the complex conjugate of that from a spontaneously emitted photon.

Our present work is also related to two recent developments. The first one is the implementation of a microwave photodetector using phase-biased Josephson junctions in Ref. [19]. This setup contains some ingredients that are needed for the proposals in this and previous papers $[15,16]$, and in particular its layout closely resembles the ones put forward in this paper. The second work is devoted to the study of the quasibound states that appear when a qubit is confronted with a mirror [20]. Those resonances are to a large extent responsible for the high efficiency and long interaction times between incoming photons and our detector. This is further evidenced in our study of photodetection when the photons are directly injected between the qubit and the mirror (Sec. IV).

Finally, we want to remark that the developments in this and previous works $[15,16]$ are very general. The formalism is based on one-dimensional waveguides and three-level systems, and it could be trivially exported to novel and fascinating experimental setups, such as single-mode fibers or photonic waveguides interacting with atoms [21], or plasmonic waves coupled to quantum dots or nitrogen-vacancy $(\mathrm{NV})$ centers 


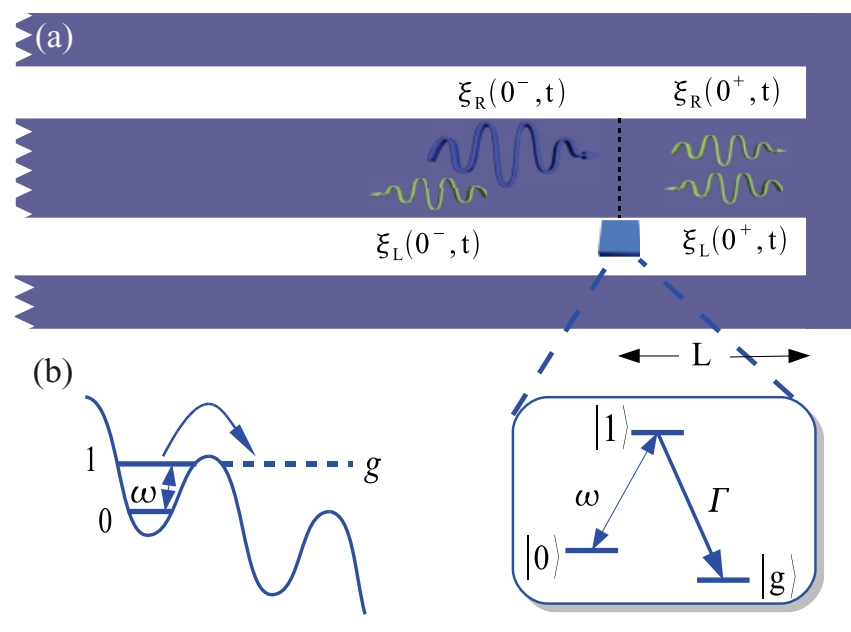

FIG. 1. (Color online) Scheme of our microwave photon detector proposal. (a) The setup consists of a metastable quantum circuit positioned at a distance $L$ from the right mirror of a one-sided cavity, forming a pseudocavity. (b) The quantum circuit can be made from a current-biased Josephson junction, in which a washboard potential confines two metastable states that can decay into a continuum of current states.

$[22,23]$, where the degree of controllability and interaction strength are expected to approach those of circuit QED in the near future.

This work is organized as follows. In Sec. II we study in detail a design that consists of a three-level system sitting on a semi-infinite transmission line and how it interacts with a finite-width propagating photon. We work in the stronginteraction, rotating-wave-approximation regime, in which the number of excitations is preserved, and develop an analytic approximation to the dynamics (Sec. II A), including a simple analyitical expression for long wave packets (Sec. II B). With these tools we can demonstrate that for a wide variety of parameters a single photon may be perfectly absorbed by the three-level detector, even when it is detuned (Sec. II C). In Sec. III we develop a simplified theory based on scattering of plane waves which reproduces the previous results and allows us to study setups with more than one three-level system. The main result is that an increased number of absorbers enhances the robustness, the bandwidth, and the overall performance of the detector. In Sec. IV we slightly modify our theory to study what happens when photons are not coming from the semi-infinite transmission line, but rather injected through the end of the line. We will show that efficient photodetection is still possible and is mediated by quasilocalized states between the qubit and the mirror, at the expense of longer detection times. Finally in Sec. V we summarize our results.

\section{A QUBIT AND A MIRROR}

In this section, we discuss the simple setup of a semi-infinite transmission line coupled to a metastable quantum circuit located at a distance $L$ from the end of the waveguide, which acts as a mirror (see Fig. 1). Studying the problem in real space, we derive the relevant equations describing the dynamics of the system. These models are used to study the scattering of a photon wave packet, showing that, under realistic conditions, it is completely absorbed by the metastable quantum circuit which represents the detector itself. More precisely, we demonstrate that when the photon is not reflected, the fraction that bounces back and forth between the absorber and the end mirror is also absorbed and thus detected. This will be the starting point for a more general and simpler theory in the following section.

\section{A. One absorber interacting with a single photon}

As sketched before, the basis of our work, as in our previous proposal $[15,16]$, is the real-space representation of a one-dimensional waveguide interacting with a single qubit [24]. The model consists of a non-Hermitian Hamiltonian that contains terms for the metastable quantum circuits or "absorbers," modeled as three-level systems, and the radiation fields $\psi_{R}$ and $\psi_{L}$ propagating to the right and to the left with group velocity $v_{g}$,

$$
\begin{aligned}
H= & \sum_{i} \hbar\left(\omega_{i}-i \frac{\Gamma_{i}}{2}\right)|1\rangle_{i}\langle 1| \\
& +i \hbar v_{g} \int\left[\psi_{L}^{\dagger} \partial_{x} \psi_{L}-\psi_{R}^{\dagger} \partial_{x} \psi_{R}\right] d x \\
& +\sum_{i} \hbar V \int \delta\left(x-x_{i}\right)\left[\left(\psi_{R}+\psi_{L}\right)|1\rangle_{i}\langle 0|+\text { H.c. }\right] d x
\end{aligned}
$$

Note how the interaction between photons and circuits is modeled using a $\delta$ potential of strength $V$ located at the positions of the latter, $x_{i}$. In this notation, $|0\rangle$ and $|1\rangle$ represent the two states of the absorber connected by the photon [see Fig. 1(b)], $\Gamma_{i}$ stands for the decay rate from the metastable state $|1\rangle$, and $\omega_{i}$ is the frequency separation between $|0\rangle$ and $|1\rangle$.

The simplest scenario that we consider is a single photon interacting with one absorber placed at $x=0$, as shown in Fig. 1(a). The photon coming from the left with energy $E=$ $\hbar|k| v_{g}$ will exchange its excitation. The most general wave function that describes this process is [24]

$$
|\Psi\rangle=\int\left[\xi_{R}(x) \psi_{R}^{\dagger}(x)+\xi_{L}(x) \psi_{L}^{\dagger}(x)\right]|0, \mathrm{vac}\rangle+e(t)|1, \mathrm{vac}\rangle .
$$

The first part of the state constitutes the most general form in which the field contains just a single photon, moving right, $\xi_{R}(x)$, or left, $\xi_{L}(x)$, with the detector or absorber in the metastable state $|0\rangle$. As soon as this photon interacts with the three-level system, there is some probability that the field excitation gets absorbed and the three-level system jumps into the unstable level $|1\rangle$. When this happens, the excited-state population of the absorber, $e(t)$, increases. We will derive the evolution equations for general wave packets, $\xi_{R, L}(x)$, but in order to compute the absorption efficiency we need to impose constraints on the bandwidth and the shape of these photons.

We want to emphasize the possibility of having input states other than (2). Consider for instance an attenuated coherent state. This basically consists on Eq. (2) plus a dominant term $\mid 0$, vac $\rangle$ and higher-order terms with two and more photons. The 
higher-order terms can be neglected and thus the absorption efficiency basically coincides with the one derived using (2). Another possibility would be an incoherent mixture of different single-photon wave packets $\rho=\int p[\xi]\left|\psi_{\xi}\right\rangle\left\langle\psi_{\xi}\right| \mathcal{D} \xi$, with some distribution over wave-packet shapes and properties, $p[\xi]$. One example of this is a statistical mixture of single photons whose emission time and phase cannot be precisely determined, as would be the case of photons emitted by a driven qubit [8] or scattered by a two-level system [25]. In this simple case the total efficiency will be the average over the possible input states. However, given that the same source will produce identical photons through time, and that our single-photon detector will be insensitive to the arrival time and to the phase (Sec. II B), we will find that it is enough to work with the ansatz (2).

Solving the Schrödinger equation with the non-Hermitian Hamiltonian (1) leads to a set of equations containing the field and absorber amplitudes:

$$
\begin{aligned}
i \partial_{t} \xi_{R}(x, t) & =-i v \partial_{x} \xi_{R}(x, t)+V \delta(x) e, \\
i \partial_{t} \xi_{L}(x, t) & =+i v \partial_{x} \xi_{L}(x, t)+V \delta(x) e, \\
\left(i \partial_{t}-\omega+i \frac{\Gamma}{2}\right) e & =\frac{V}{2}\left[\xi_{R}^{+}+\xi_{R}^{-}+\xi_{L}^{+}+\xi_{L}^{-}\right],
\end{aligned}
$$

where we abbreviate $\xi_{R, L}^{ \pm}(t):=\xi_{R, L}\left(0^{ \pm}, t\right)$. As explained elsewhere [16], our quantum jump description allows us to compute the population of the level $|g\rangle$ as $P_{g}=1-\|\Psi\|^{2}$. Indeed, the value of $P_{g}$ at long times is what we call the detector efficiency and can be fully determined from the previous equations, after a few manipulations.

Note that two equations in (3) can be turned into boundary conditions around the absorber

$$
\xi_{R}^{+}=\xi_{R}^{-}-i \frac{V}{v_{g}} e, \quad \xi_{L}^{-}=\xi_{L}^{+}-i \frac{V}{v_{g}} e .
$$

This allows us to express the amplitude of the unstable state $|1\rangle$ in terms of the left and right incoming fields, that is,

$$
\left[i \partial_{t}-\omega+i \frac{\Gamma}{2}+i \frac{V^{2}}{v_{g}}\right] e=V\left[\xi_{R}^{-}+\xi_{L}^{+}\right] .
$$

The above procedure is standard in any single-photon scattering problem, but in this case the mirror to the right end imposes another key boundary condition, which is a coupling between right- and left-propagating fields. More precisely, the only independent variable will be the field coming from the left, $\xi_{R}\left(0^{-}, t\right)=\phi(t)$, since the incoming field from the right, $\xi_{L}\left(0^{+}, t\right)$, is generated by the former, after being reflected by the mirror and affected by a phase factor $\kappa$. In other words,

$$
\xi_{L}^{+}(t)=\kappa \xi_{R}^{+}(t-a)=\kappa \phi(t-a)-\kappa i \frac{V}{v_{g}} e(t-a),
$$

with $a=2 L / v_{g}$ depending on the distance between the absorber and the mirror and the group velocity of the photons. This boundary condition provides us with a closed delay differential equation (DDE) for the amplitude of state $|1\rangle$,

$$
\begin{aligned}
i \partial_{t} e(t)= & {\left[\omega-i \frac{\Gamma}{2}-i \frac{V^{2}}{v_{g}}\right] e(t)-\kappa i \frac{V^{2}}{v_{g}} e(t-a) } \\
& +V \phi(t)+\kappa V \phi(t-a),
\end{aligned}
$$

thereby specifying the complete dynamics of the system for any incoming signal.

\section{B. Adiabatic limit}

DDEs are very complicated mathematical objects which rarely have analytic solutions and which typically lead to nonlinear phenomena. In order to simplify the treatment, avoid critical behavior, and get some understanding of the detection of realistic wave packets, we will make some additional simplifications. More precisely, we will assume an incoming wave packet with frequency $\omega_{0}$ and phase $\eta$ and adiabatically modulate

$$
\phi(t)=\chi(t) \exp \left(-i \omega_{0} t+i \eta\right), \quad\left|\partial_{t} \chi\right| \ll \omega_{0} .
$$

This ansatz has various consquences for the dynamics. First of all, the absorber itself will evolve according to the main frequency, $e(t)=v_{g} x(t) \exp \left(-i \omega_{0} t+i \eta\right) / V$. Second, introducing the constants $\theta=\omega_{0} a$ and $a=v_{g} \Delta / V^{2}$ and making the change of variables $t=v \tau / V^{2}$, we will obtain a simplified equation

$$
\begin{aligned}
i \partial_{\tau} x(\tau)= & -i(1+\gamma) x(\tau)-i z x(\tau-\Delta) \\
& +\chi(\tau)+z \chi(\tau-\Delta),
\end{aligned}
$$

with only two free parameters

$$
\gamma=\frac{v_{g}}{V^{2}}\left[\frac{\Gamma}{2}+i\left(\omega-\omega_{0}\right)\right], z=\kappa e^{i \theta} .
$$

Finally, using the adiabatic approximation, that is, the smoothness of the envelope, $\left|\partial_{\tau} \chi\right| \ll \omega_{0}$, we may replace $\chi(\tau-\Delta)$ by $\chi(\tau)$, and integrate the resulting equation

$$
x(\tau)=-i(1+z) \int_{-\infty}^{\tau} e^{-(1+\gamma+z)(\tau-s)} \chi(s) d s .
$$

The whole problem has simplified considerably, and in particular the dependency on the global phase $\eta$ has disappeared completely.

\section{Test wave packets}

Starting from expression (11) we would like to compute the efficiency of the detector. The integral in that equation is roughly a Fourier transform of the adiabatic modulation, and we expect that the the left-hand-side term, in the limit $t \rightarrow \infty$, does not depend much on the fine details of the driving field. One may now study, for instance, a normalized Gaussian wave packet

$$
\chi(\tau)=\frac{1}{\sqrt{\sigma \sqrt{\pi}}} \exp \left[-\tau^{2} /\left(2 \sigma^{2}\right)\right],
$$

and how it is scattered by the three-level system. The Gaussian form is chosen for convenience, but it is in no way essential for the results. This Gaussian has the advantage that in the limit $\sigma \rightarrow \infty$ it contains the case of infinite plane waves, a limit which we used in previous works and which we would like to recover. However, as long as the wave packet remains adiabatic, that is, $\sigma \gg \omega_{0}^{-1}$, none of the results will depend dramatically on its precise shape, as we confirmed numerically.

We are now in a position to compute the transmitted and reflected wave packets $\xi_{R}^{+}(t), \xi_{L}^{-}(t)$, the dynamics of the 

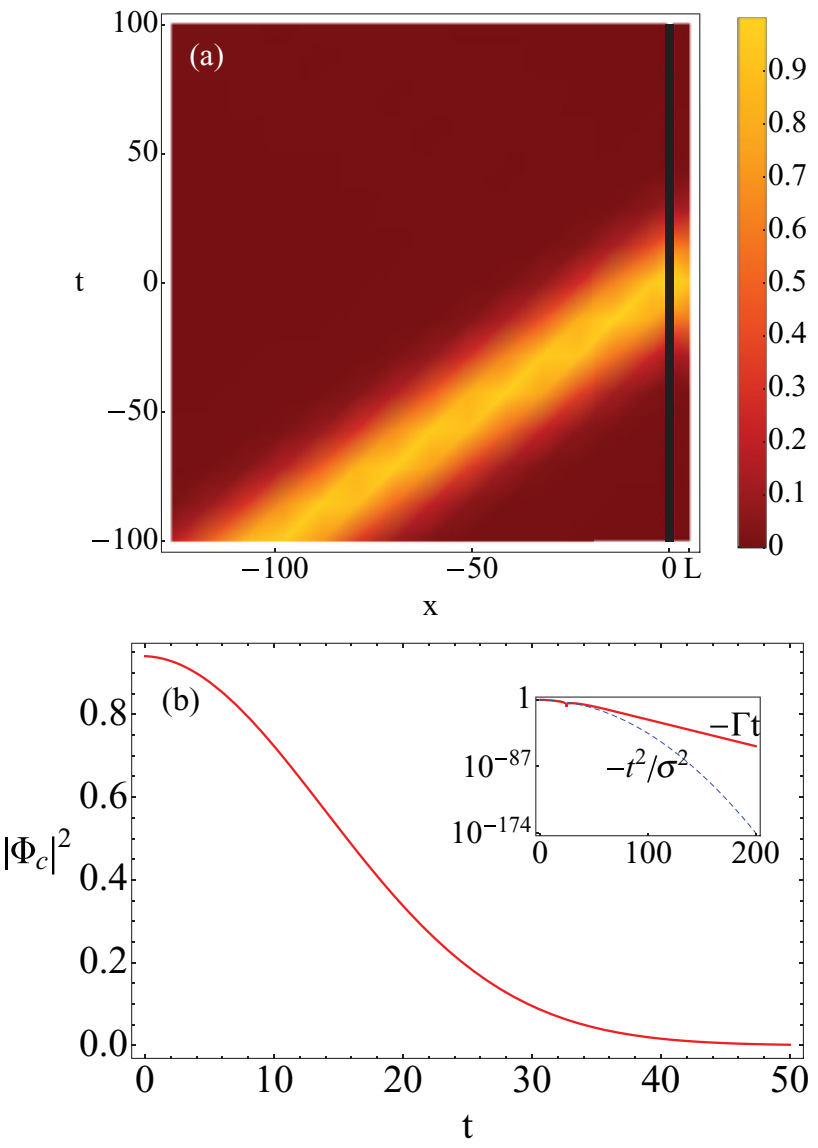

FIG. 2. (Color online) (a) Time evolution of an incident wave packet that undergoes no reflection, leading to a confined field to the right of the absorber. (b) Decay of the field inside the absorber-mirror cavity. The inset shows a logarithmic plot of the field amplitude, exhibiting a time scale with decay.

detector $x(t)$, and even the detection probability mentioned before, all as a function of the parameters $\sigma, \gamma$, and $z$. The first result that we show in Fig. 2(a) is that there indeed exist configurations for which no incoming photon is reflected. In such setups the photon tunnels through the qubit and bounces back and forth between the qubit and the mirror. If this process continued indefinitely, our system could not perform as a photodetector, as it would never capture the photon and switch. In order for the photodetection to succeed, the three-level system must be able to absorb the confined field completely and undergo an irreversible transition to the "click" state $|g\rangle$. Fortunately, as Fig. 2(b) shows, the population of the field inside the qubit-mirror pseudocavity dissipates very quickly, and in a time scale determined by the decay channel of the absorber, $\Gamma^{-1}$, the absorber fully detects the confined photon.

The above results give us a hint that a single absorber with a mirror could reach $100 \%$ detection efficiency, but this result must be confirmed for a larger variety of experimental parameters. In order to make the study more systematic, we introduce the detector efficiency as the fraction of the wave packet that was absorbed, given by

$$
\alpha=1-\frac{\int_{-\infty}^{\infty}\left|\xi_{L}\left(0^{-}, t\right)\right|^{2}}{\int_{-\infty}^{\infty}\left|\xi_{R}\left(0^{-}, t\right)\right|^{2}} .
$$

This value is computed numerically for different photon profiles $\sigma$ and varying setup parameters $\omega, \omega_{0}, \theta$, and $\Gamma$. With respect to the pulse width, we have found that any value of $\sigma>10$ gives approximately the same result. For the other parameters we have to distinguish the resonant and nonresonant cases, and in the latter study the dependence of the efficiency on the detuning, $\delta=\omega-\omega_{0}$. As shown in Fig. 3(a), for a resonant incident photon the efficiency reaches a maximum of $100 \%$ around $\theta=\pi$ and $\Gamma=2$ [Fig. 3(a)], where $\Gamma$ is in units of $V^{2} / v_{g}$. When the photon is off resonant, $\delta \neq 0$, we obtain two remarkable results. First of all, theoretical perfect detection is still possible, and second, this happens for two different sets of parameters, as shown in Fig. 3(b). The relative position of the two maxima depends on the coupling strength $V$. These solutions approach each other [Fig. 3(c)] until the detuning reaches a threshold $\delta \leqslant V^{2} / v_{g}$, where the two solutions merge and disappear. Using parameters in the range used in Ref. [15], this sets the limit of the bandwidth around $\delta \sim 10-100 \mathrm{MHz}$ for just a single detector, but it increases for larger couplings.

\section{SCATTERING THEORY}

In the previous section we demonstrated two important results. The first one is that the scattering of a realistic wave packet through a single three-level system indicates the existence of a regime of theoretically perfect photodetection. The second one is that we can analytically compute all scattering properties for a sufficiently large wave packet and that these values are almost insensitive to the wave-packet size. This result motivates us to replace the previous formalism with a simpler one, based on the scattering of plane waves through one or multiple three-level systems. This method, developed in Ref. [24] and applied in our photodetector works [15,16], has the advantage that it scales well to setups with multiple detectors, an ingredient which is crucial for enhancing the robustness and the bandwidth of the detector.

Consider an incident monochromatic beam interacting with more qubits, using the scattering theory developed in Refs. [15, 16,24]. The idea is that the fields on the left and on the right of the absorbers are related by a scattering matrix

$$
\left(\begin{array}{c}
\xi_{R}^{\prime} \\
\xi_{L}^{\prime}
\end{array}\right)=T\left(\begin{array}{l}
\xi_{R} \\
\xi_{L}
\end{array}\right)
$$

where $T$ stands for the transfer matrix and takes the form

$$
T=\prod_{j}^{N} e^{i \frac{2 \pi L_{j}}{\lambda} \sigma_{z}} T_{j}, \quad T_{j}=\left(\begin{array}{cc}
1-1 / \gamma & -1 / \gamma \\
1 / \gamma & 1+1 / \gamma
\end{array}\right) .
$$

Compared to Ref. [15], the main difference now is that after leaving the scatterers and confronting the mirror, the field has to satisfy a boundary condition

$$
\left(\begin{array}{c}
1 \\
\kappa
\end{array}\right)=\exp \left(i \theta \sigma^{z}\right) T\left(\begin{array}{l}
\xi_{R} \\
\xi_{L}
\end{array}\right) .
$$

The parameter $\theta$ is the phase acquired by the photon between the last scatterer and the mirror, while $\kappa$ is the boundary condition for the mirror to have zero field, typically -1 . The previous equations hide a relation between the incoming 

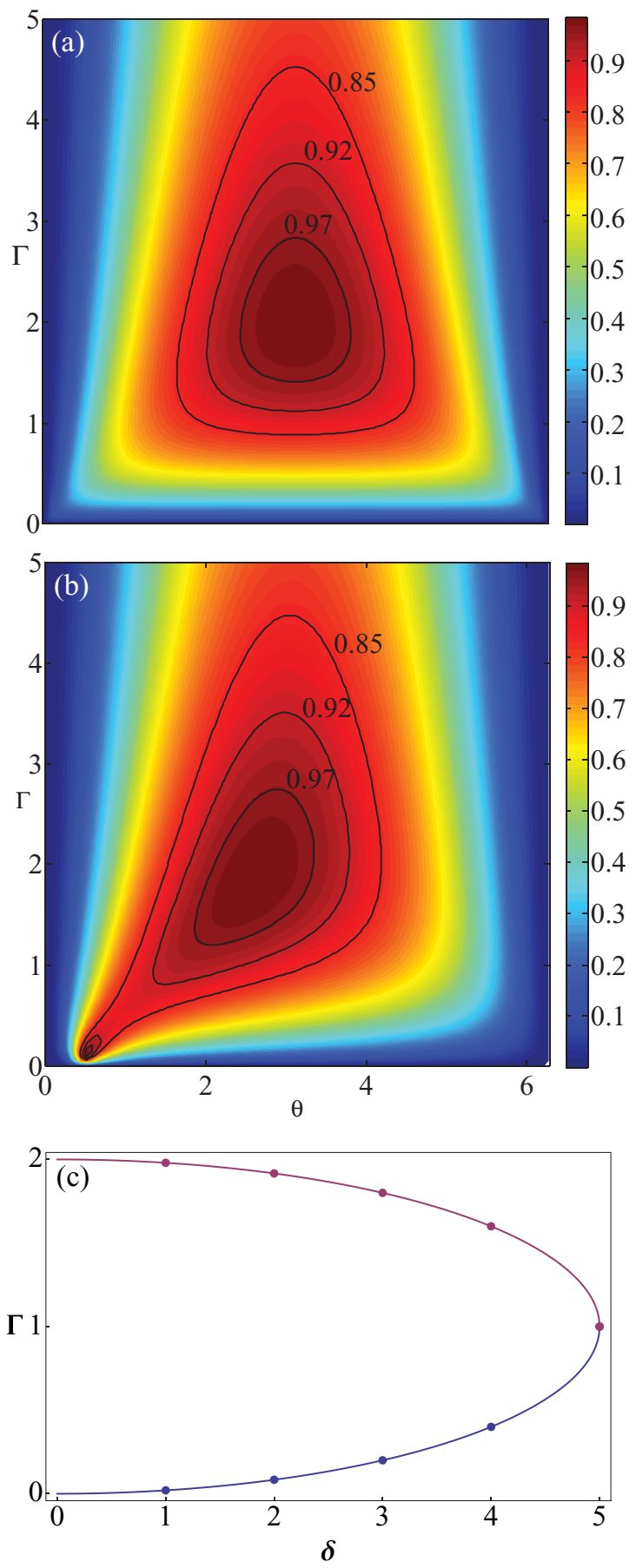

FIG. 3. (Color online) (a) Detection efficiency as a function of the decay $\gamma$ and the phase $\theta$ when the three-level system is on resonance with the incident photon. (b) Real part of the detection efficiency for the off-resonance case. (c) Position of the efficiency maxima as a function of the detuning (off-resonance case).

field $\xi_{R}^{-}$and the reflected one $\xi_{L}^{-}$, which can be revealed by projecting onto an orthogonal subspace

$$
(\kappa-1) \exp \left(i \theta \sigma^{z}\right) T\left(\begin{array}{l}
\xi_{R} \\
\xi_{L}
\end{array}\right)=0
$$

In the case of a single absorber $(N=1)$, we directly obtain an analytic expression for the outgoing field,

$$
\xi_{L}=\left[\frac{\gamma(z+1)}{1+\gamma+z}-1\right] \xi_{R},
$$

which becomes exactly zero for

$$
\gamma=1+z^{-1},
$$

reflecting the limit in which no photon is reflected and all photons are absorbed, in perfect agreement with the exact results for Gaussian wave packets developed in the previous section.

Using this formalism, we can go beyond one absorber, studying the optical properties of a setup with multiple threelevel systems in front of a mirror. Inspired by our previous works we expect that a setup with multiple scatterers will help, first, by increasing the robustness of the detector and, second, by enlarging the band of frequencies for which almost perfect detection takes place. Furthermore, as shown in those works, the way in which we place the absorbers is very relevant, as placing them too close together does not have any influence in the detector efficiency or bandwidth. For simplicity, we will adopt the optimal configuration from the open line, with equally spaced absorbers. From the elements of the transfer matrix given by Eq. (15), we can compute the absorption efficiency (13) using the formula

$$
\alpha=1-\left|\frac{T_{11}+e^{i \theta} T_{12}}{T_{21}+e^{i \theta} T_{22}}\right|^{2},
$$

where now $T_{i j}$ depends on the number of absorbers, $N$, and the previous two parameters $\Gamma$ and $\theta$.

As an illustration, in Fig. 4 we show three plots that demonstrate the enhanced bandwidth and decreased sensitivity to the qubit and setup properties $\Gamma$ and $\theta$. To start with, let us look at Fig. 4(a), which plots the detector efficiency for $N=4$ absorbers. Compared with Fig. 3(a), the maximum efficiency is extended to a larger region of mirror separations, now centered around $\pi / 2,3 \pi / 2$, and tolerates also a larger set of decay rates $\Gamma$. This is further confirmed when we study the evolution of the efficiency for increasing number of absorbers. For instance, Fig. 4(b) represents the efficiency as a function of the phase $\theta=4 \pi L / \lambda$, where $L$ stands for the distance between absorbers. Notice that, for $N=8$ absorbers, the efficiency reaches more than $90 \%$ almost independently of $\theta$, the relative position between absorbers becoming less important. A similar effect happens with the detuning, and as Fig. 4(c) shows, the set of multiple detectors very quickly acquires a large bandwidth, even faster than in our previous works $[15,16]$.

\section{DETECTING THROUGH THE MIRROR}

On looking at our setup a natural question arises: what happens if the photon is not coming from the semi-infinite transmission line, but instead it "tunnels" through the mirror, which is not perfect? This is an interesting question for a number of reasons. The first one is that if the photon is directly injected between the mirror and the cavity it has a great chance to probe quasibound states existing between both, providing further evidence that the qubit and the mirror form 

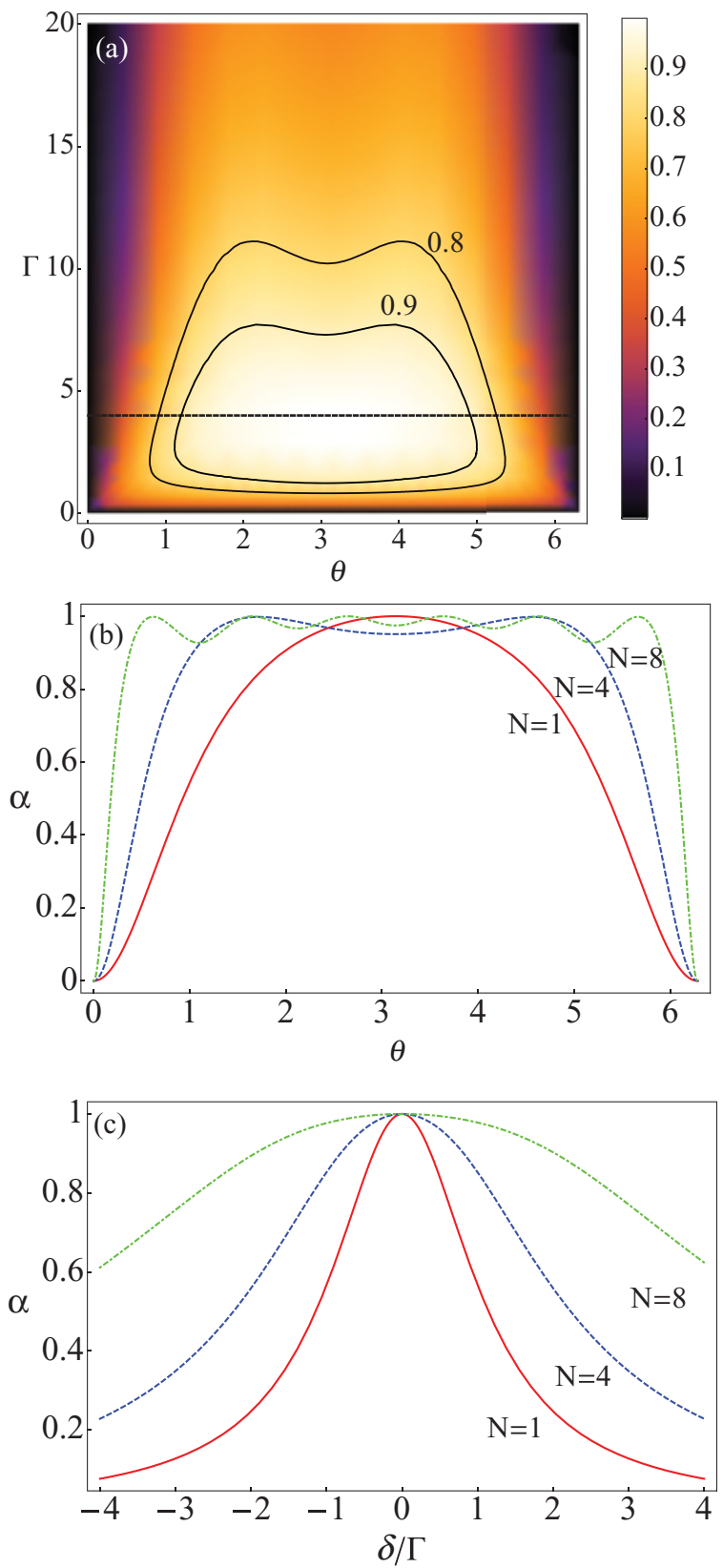

FIG. 4. (Color online) (a) Absorption efficiency for four qubits as a function of the decay and phase $\theta$. (b) Performing a cut along the optimal value of $\Gamma$ (dashed line), we compute the efficiency as a function of $\theta$ for $N=1,4$, and 8 absorbers. (c) Efficiency dependence on the detuning rate

a pseudocavity [20]. The second reason is that this setup is close to the recent experiment [19] which demonstrates the photodetection capabilities of a phase-biased junction.

Describing a semi-infinite line with an imperfect mirror would severely depart from the methods introduced in this paper, requiring the introduction of environments, decoherence, and master equations. Fortunately, there is a simple "toy" model that contains the essential ingredients of the problem and which can still be treated with the scattering formalism. In our model the photon is directly tunneling between the qubit and the mirror, as shown in Fig. 5(a), and the only way it

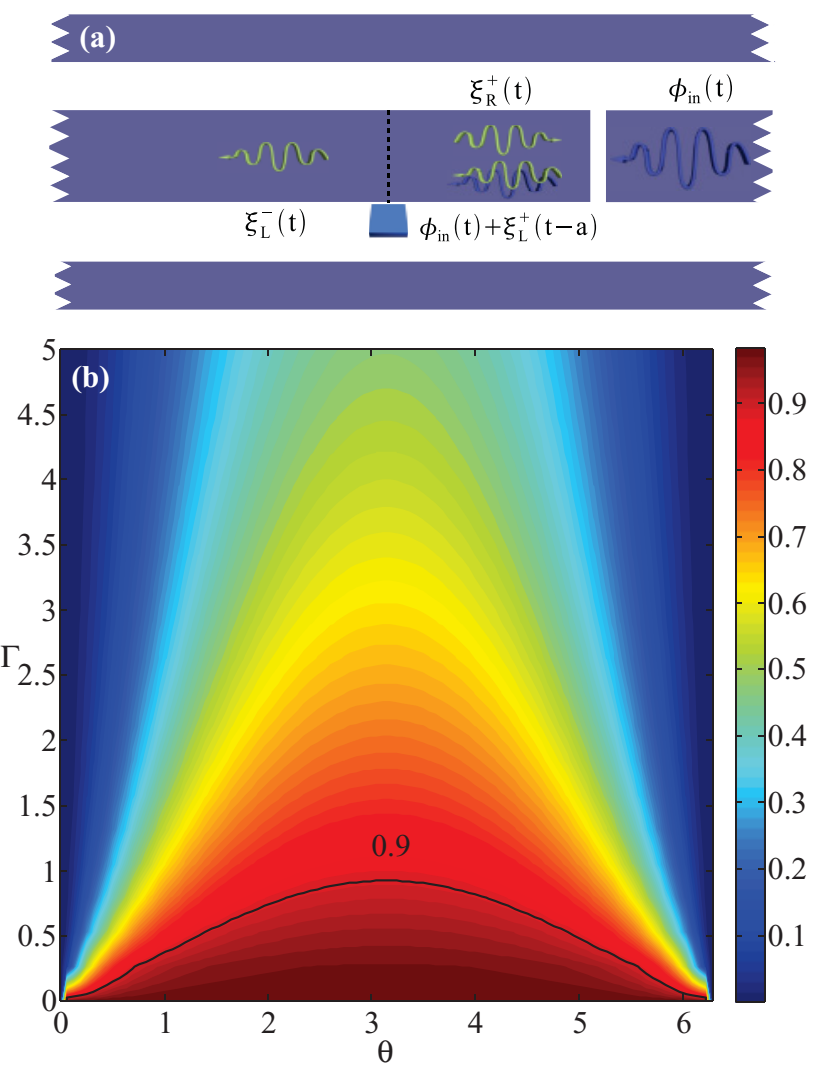

FIG. 5. (Color online) (a) Photodetector scheme for an incident photon coming from the right. (b) Detection efficiency associated with this detector.

may leak is by passing through the qubit again. The incoming photon profile will be denoted by $\phi_{i n}(t)$, and we expect that the wave packet is partially trapped into the pseudocavity formed by the mirror and the absorber and is partially transmitted. Working with our previous single-photon formalism we obtain the following set of equations for the field amplitudes:

$$
\begin{aligned}
& \xi_{R}^{-}(t)=0, \quad \xi_{R}^{+}(t)=\xi_{R}^{-}(t)-i \frac{V}{v_{g}} e(t), \\
& \xi_{L}^{-}(t)=\xi_{L}^{+}(t)-i \frac{V}{v_{g}} e(t), \quad \xi_{L}^{+}(t)=\phi_{i n}(t)+\kappa \xi_{R}^{+}(t-a),
\end{aligned}
$$

where the presence of the time-delayed amplitude field $\xi_{R}^{+}$is due to the iterative feedback with the mirror. Using the same tools, we can now compute the detection efficiency associated with the switching process of the three level system. As shown in Fig. 5(b), the contour plots of the efficiency suffer a radical change. While the maxima are still at a resonant distance between mirror and qubit, the $100 \%$ detection efficiency is strictly achieved for $\Gamma \rightarrow 0$.

The previous analytical results have a very clear interpretation. In order to have a large detection efficiency, the photon has to spend a long time bouncing between the mirror and the qubit. However, as shown previously [24], the qubit acts as a perfect mirror only strictly for $\Gamma=0$. The consequence is that in this setup the decay time of the three-level system, $\Gamma$, must 
approach zero to increase the efficiency, and at the same time the detection time diverges as $1 / \Gamma$. In other words, while this setup seems quantitatively similar to the previous ones, it does not work in practice, because, first, the tunneling probability of the photon through the mirror will be small and, second, the detection times are so long that the process will be damaged by decoherence and losses.

\section{SUMMARY AND CONCLUSIONS}

The main result of this work is that a three-level system, implemented as a phase qubit, a biased Josephson junction, or whatever seems adequate, is an almost perfect photodetector. The efficiency of this device is limited only by how it couples to the waves that contain the photons it has to detect. In particular, it seems that the setup we originally proposed $[15,16]$ can be improved by replacing a completely open transmission line with a semi-infinite line that brings the photons to the detector, allowing repeated interactions. We have studied this absorber-mirror system in detail. Instead of a simple scattering model $[15,16]$, we now have a delay differential equation that allows for nonlinear behavior and in particular for a constructive interference effect. We have demonstrated that, thanks to this effect, when an incoming photon passes through the only qubit without reflection it is actually absorbed. This first result is quite important, because it rules out that the photon gets trapped in a metastable confined state between the absorber and the mirror [19], and because it allows us to develop a much simplified theory based on the scattering of plane waves. With this theory we confirmed the $100 \%$ efficiency of a single absorber, and extended our design to include multiple qubits in front of a mirror, a setup that shows enhanced bandwidth and very much decreased sensitivity to the detector properties.

The insensitivity of the detector to the phase and its large bandwidth can be used to prove numerically, as in Ref. [16], its robustness against imperfections in the qubit specifications and dephasing, which are the most important sources of decoherence. Furthermore, since we use the same ingredients as in our previous proposal, all other considerations about robustness still apply. For instance, spontaneous decay from the 1 to the 0 level is also not a problem, because this mainly happens through radiative decay in which the qubit switches its state and deposits a photon on the line. But, as we have seen above, this decay process is contemplated in the theory through the coupling "V": in other words, spontaneous emission is part of what makes the detector work. We can also neglect photon losses, nonradiative qubit decay, and leaky mirrors, because they operate on time scales which are much longer than the photon wave packet. Finally, the greatest technical difficulty of the setup is the control of the three-level system, to avoid dark counts, that is, spontaneous transitions of the phase qubit from the 0 state to the " $g$ " states. This can be dealt with by controlling the ratio between decay rates of the 0 and the 1 levels to the continuum. As is currently done with phase qubits, by tuning the bias it is possible make the jump probability of $0 \rightarrow g$ a thousand times smaller than $\Gamma$, which is typically longer than the photon wave packets considered here. Another possibility is to periodically refresh the detector, resetting it to the 0 state, similar to the periodic refreshing that happens with some photodetectors in the optical regime.

Overall, a small change in the photodetector design has been shown to provide an enormous increase in detection efficiency, revealed a different dynamics with a great potential for further development, while making the setup simpler and much more attractive for actual implementation. We strongly believe that this setup is now suitable for integration in ongoing circuit QED experiments with two-level systems and single photons [8,9,25-27]. In this case one might need to use circulators to prevent reflection from the detector and mutual interactions spoiling the emitter signal. A realistic example is found in Ref. [25], where the photons scattered by a two-level system are shown to have widths of about $1 \mathrm{~ns}$ (a few centimeters) and can be efficiently routed by circulators on the same chip.

Finally, we expect that many of the ideas in this work can be translated to other physical systems that combine fiber, photonic, or plasmonic waveguides with atoms, quantum dots or NV centers [21-23], once the current degree of controllability and interaction strength of circuit QED is achieved.

\section{ACKNOWLEDGMENTS}

We thank Anders Sørensen for pointing out the relation between our work and Refs. [18,28], and the discussions that sparked this research. B.P. thanks Jordi Mur-Petit for useful discussions. This work was supported by Spanish MICINN Project No. FIS2009-10061, and CAM research consortium QUITEMAD Project No. S2009-ESP-1594. B.P. acknowledges funding by a CSIC JAE-PREDOC2009 Grant. G.R. acknowledges funding from the Juan de la Cierva program. G.J. and C.M.W. thank the Swedish Research Council (VR) and European Research Council for funding. E.S. acknowledges funding from Basque Government Grant No. IT472-10, Spanish MICINN Project No. FIS2009-12773-C02-01, the SOLID European project, and the EU CCQED project.
[1] Y. Nakamura, C. D. Chen, and J. S. Tsai, Phys. Rev. Lett. 79, 2328 (1997).

[2] V. Bouchiat, D. Vion, P. Joyez, D. Esteve, and M. H. Devoret, Phys. Scr., T 76, 165 (1998).

[3] J. E. Mooij, T. P. Orlando, L. Levitov, L. Tian, C. H. van der Wal, and S. Lloyd, Science 285, 1036 (1999).
[4] J. M. Martinis, S. Nam, J. Aumentado, and C. Urbina, Phys. Rev. Lett. 89, 117901 (2002).

[5] A. Blais, R.-S. Huang, A. Wallraff, S. M. Girvin, and R. J. Schoelkopf, Phys. Rev. A 69, 062320 (2004).

[6] A. Wallraff, D. Schuster, A. Blais, L. Frunzio, R.-S. Huang, J. Majer, S. Kumar, S. M. Girvin, and R. J. Schoelkopf, Nature (London) 431, 162 (2004). 
[7] I. Chiorescu, P. Bertet, K. Semba, Y. Nakamura, C. J. P. M. Harmans, and J. E. Mooij, Nature (London) 431, 159 (2004).

[8] D. Bozyigit et al., Nat. Phys. 7, 154 (2011).

[9] C. Eichler, D. Bozyigit, C. Lang, L. Steffen, J. Fink, and A. Wallraff, Phys. Rev. Lett. 106, 220503 (2011).

[10] F. Mallet, M. A. Castellanos-Beltran, H. S. Ku, S. Glancy, E. Knill, K. D. Irwin, G. C. Hilton, L. R. Vale, and K. W. Lehnert, Phys. Rev. Lett. 106, 220502 (2011).

[11] M. Mariantoni, M. J. Storcz, F. K. Wilhelm, W. D. Oliver, A. Emmert, A. Marx, R. Gross, H. Christ, and E. Solano, e-print arXiv:cond-mat/0509737.

[12] M. Mariantoni, E. P. Menzel, F. Deppe, M. A. Araque Caballero, A. Baust, T. Niemczyk, E. Hoffmann, E. Solano, A. Marx, and R. Gross, Phys. Rev. Lett. 105, 133601 (2010).

[13] E. P. Menzel, F. Deppe, M. Mariantoni, M. A. Araque Caballero, A. Baust, T. Niemczyk, E. Hoffmann, A. Marx, E. Solano, and R. Gross, Phys. Rev. Lett. 105, 100401 (2010).

[14] E. Knill, R. Laflamme, and G. J. Milburn, Nature (London) 409, 46 (2001).

[15] G. Romero, J. J. García-Ripoll, and E. Solano, Phys. Rev. Lett. 102, 173602 (2009).

[16] G. Romero, J. J. García-Ripoll, and E. Solano, Phys. Scr. 2009, 014004 (2009).

[17] F. Helmer, M. Mariantoni, E. Solano, and F. Marquardt, Phys. Rev. A 79, 052115 (2009).
[18] D. Pinotsi and A. Imamoglu, Phys. Rev. Lett. 100, 093603 (2008).

[19] Y. Chen, D. Hover, S. Sendelbach, L. Maurer, S. T. Merkel, E. J. Pritchett, F. K. Wilhelm, and R. McDermott, Phys. Rev. Lett. 107, 217401 (2011).

[20] H. Dong, Z. R. Gong, H. Ian, L. Zhou, and C. P. Sun, Phys. Rev. A 79, 063847 (2009).

[21] E. Vetsch, D. Reitz, G. Sagué, R. Schmidt, S. T. Dawkins, and A. Rauschenbeutel, Phys. Rev. Lett. 104, 203603 (2010).

[22] D. E. Chang, A. S. Sørensen, P. R. Hemmer, and M. D. Lukin, Phys. Rev. Lett. 97, 053002 (2006).

[23] A. V. Akimov, A. Mukherjee, C. L. Yu, D. E. Chang, A. S. Zibrov, P. R. Hemmer, H. Park, and M. D. Lukin, Nature (London) 450, 402 (2007).

[24] J.-T. Shen and S. Fan, Phys. Rev. Lett. 95, 213001 (2005).

[25] I.-C. Hoi, C. M. Wilson, G. Johansson, T. Palomaki, B. Peropadre, and P. Delsing, Phys. Rev. Lett. 107, 073601 (2011).

[26] O. Astafiev, A. M. Zagoskin, A. A. Abdumalikov, Y. A. Pashkin, T. Yamamoto, K. Inomata, Y. Nakamura, and J. S. Tsai, Science 327, 840 (2010).

[27] A. A. Abdumalikov, O. V. Astafiev, Y. A. Pashkin, Y. Nakamura, and J. S. Tsai, Phys. Rev. Lett. 107, 043604 (2011).

[28] D. Witthaut and A. S. Sørensen, New J. Phys. 12, 043052 (2010). 